Published in Phys.Rev.B 83, 035411 (2011)

\title{
Polarization effects in Non-Contact Atomic Force Microscopy: a key to model the tip-sample interaction above charged adatoms
}

\author{
Franck Bocquet ${ }^{1,2}$, Laurent Nony ${ }^{1,2}$, and Christian Loppacher ${ }^{1,2} *$ \\ (1) Aix-Marseille Université, IM2NP, \\ Centre Scientifique de Saint-Jérôme, \\ Avenue Escadrille Normandie-Niemen, Case 151, \\ F-13397 Marseille CEDEX 20, France \\ ${ }^{(2)}$ CNRS, IM2NP (UMR 6242), Marseille-Toulon, France
}

(Dated: January 26, 2011)

* To whom correspondence should be addressed; E-mail: Christian.Loppacher@im2np.fr. 


\begin{abstract}
We discuss the influence of short-range electrostatic forces, so called dipolar forces, between the tip of an atomic force microscope (AFM) and a surface carrying charged adatoms. Dipolar forces are of microscopic character and have their origin in the polarizability of the foremost atoms on tip and surface. In most experiments performed by non-contact AFM, other forces such as binding forces dominate the interaction. However, in the experiments presented by Gross et al. [Science 324, 1428 (2009)], where the charge state of individual gold atoms adsorbed on a thin dielectric layer was determined, binding forces are negligible as the tip-sample distance is relatively large.

We develop a model which mimics the experimental tip-sample geometry of the aforementioned experiments. The model includes van der Waals and long-range electrostatic interactions, as well as the short-range electrostatic interaction based on the self-consistent description of electronic polarization effects on neutral and charged adatoms. The model is based on a calculation of the electrostatic energy of the tip-sample geometry.

Our calculations of non-contact AFM imaging as well as of bias spectroscopic curves are in good agreement with the experimental ones presented by Gross et al. It is demonstrated that the short-range dipolar force is mainly responsible for the contrast observed in topography imaging above charged species. However, it is the long-range capacitive force which is responsible for the detection of the charge state in bias spectroscopy. We discuss implications of our findings on future experiments which aim to detect single charges by means of Kelvin probe force microscopy.
\end{abstract}

PACS numbers: 07.79.Lh, 41.20.Cv, 34.20.-b

Keywords: noncontact-AFM; atomic resolution; short-range forces; electronic polarization; charge identification 


\section{INTRODUCTION}

Atomic force microscopy $(\mathrm{AFM})^{1}$ in the so called non-contact mode (nc-AFM) ${ }^{2,3}$ was used for the first time by Giessibl ${ }^{4}$ to measure the semiconducting surface of $\mathrm{Si}(111) 7 \mathrm{x} 7$ with atomic resolution. Since then, nc-AFM developed to an almost standard tool for imaging not only semiconducting, but also metallic and insulating surfaces with atomic resolution (cf. Ref.[5]). Although nc-AFM imaging with atomic resolution is now routinely achieved, there is still a debate about the microscopic origin of the contrast formation.

For semiconducting surfaces there is an agreement on the fact that the contrast is basically obtained due to the covalent bonding between the foremost tip atom and the surface atoms. For semiconducting surfaces with well known adsorbates, the distance dependence of these covalent binding forces can, for example, be used in comparison with theoretical calculations to chemically identify the adsorbed atom species ${ }^{6}$. In other experiments with very small tipsample separation, the angular component of the binding forces contributes to the contrast formation $^{7,8}$, which results in subatomic features in the images. However, also for larger tip-sample separations, atomic scale contrast has been observed on $\mathrm{Si}(111) 7 \mathrm{x} 7 \mathrm{Ref}$.[9]. In this work, the authors use a non-reactive oxidized silicon tip and explain that the tip-sample interaction is dominated by the dipole moment induced in the tip due to the surface charge distribution and not by covalent bonding.

For metal surfaces, the origin of the atomic contrast in nc-AFM imaging is less clear. Some works point out that for both, a clean and thus reactive silicon tip as well as for a tip contaminated by a metal atom, the tip-sample interaction would be dominated by strong covalent binding. However, this can not explain several experimental results where only weak orbital overlap was suggested and the decay length of the force was two times larger above the surface than for the tunneling current ${ }^{10,11}$. These results could only be modelled by using a tip carrying a water molecule, which resulted in only weak interaction due to van der Waals (vdW) forces, dipole-dipole interactions, and charge transfer ${ }^{12}$.

In the case of insulating surfaces, the interaction forces are multifold and strongly depend on the system under investigation. Most of the work done on insulators concentrate on two main issues: the polarity of the tip as well as the influence of dipolar interaction. It was Giessibl that first proposed an electrostatic imaging mechanism for ionic crystals based on a polarizable tip ${ }^{13}$. The proposed decay length of the interaction force in this model is 
about one order of magnitude per $\AA$ and thus sufficiently small to explain atomic scale contrast on ionic surfaces. In a series of papers using different model tips on ionic as well as oxide surfaces, Shluger et al. point out the importance of the polarization of both, tip and sample surface ${ }^{14-18}$. These extensive calculations consider, in particular, polarization effects; however, it seems difficult to separate them from the different other interactions also described in these works. The polarization is induced by the electric field of the tip. The most prominent effect due to a polar tip is the local polarization of the surface which goes along with (vertical) displacements of surface atoms. The importance of knowing the polarity of the tip when interpreting the imaging mechanisms on ionic surfaces has been discussed in detail by different groups ${ }^{19,20}$.

When it comes to imaging of local charges and surface potentials, Kelvin probe force microscopy $(\mathrm{KPFM})^{21,22}$ is the method used in most experimental setups. KPFM is a method working in combination with nc-AFM and which is based on the minimization of the electrostatic interaction between the tip and the sample. Electrostatic tip-sample interactions have been widely studied in the long-range regime on the experimental and on the theoretical level ${ }^{23-29}$ within the frame of nc-AFM and $\mathrm{KPFM}^{30-34}$. Long-range electrostatic forces cause capacitive forces connected to the capacitance of the tip/counter-electrode capacitor as well as Coulombic forces connected to the presence of charges within it.

For conducting surfaces it is well established, that KPFM reveals the so called local contact potential difference (LCPD) with a lateral resolution in the order of the tip front end $^{35-40}$. For insulating surfaces and nanoscale contrast, the contrast mechanisms are much more complex since not only the exact geometry of the tip-sample system has to be considered, but also charges and (induced) dipoles within the system (polarization effects) ${ }^{41-43}$.

Electrostatic forces in the short-range regime are known to occur since atomicallyresolved LCPD images have already been reported on semi-conducting surfaces: $\operatorname{Si}(111) 7 \times 7$ Refs.[31,44-47], $\operatorname{Si}(111) 5 \sqrt{3} \times 5 \sqrt{3}$-Sb Ref.[48], GaAs(110) Ref.[49] and InSb(001) Ref.[50], and on two bulk dielectric surfaces: $\mathrm{TiO}_{2}(110)$ Refs.[41,42] and $\mathrm{KBr}(001)$ Ref.[43]. However, the microscopic origin of these short-range electrostatic forces and the way they are coupled to the nc-AFM/KPFM imaging remains unclear, which has triggered intensive experimental and theoretical efforts in that field. Some groups initiated theoretical studies on the contribution of short-range electrostatic (SRE) forces ${ }^{43,47,50,51}$ in nc-AFM/KPFM combined experiments. In a recent series of papers ${ }^{43,52,53}$, Loppacher et al. have detailed how SRE 
forces may develop between a biased metallic tip carrying an atomic asperity such as a single atom or a small ionic cluster and the (001) facet of a bulk alkali halide single crystal. The results point out that these originate from the dynamic polarization of the atomic cluster and of surface atoms owing to the combined influence of the biased tip and of the Madelung surface potential of the ionic crystal ${ }^{13}$. In Ref.[47], Sadewasser et al. have shown that polarization effects were responsible for the atomic scale KPFM contrast on the $\mathrm{Si}(111)-7 \times 7$ including $\mathrm{Pb}$ adatoms, as well. Their results point toward a relation between the changes in the LCPD over distinct atomic sites and the variations of the surface local electronic structure due to a charge polarization induced by the tip-surface interaction. More recently, Bieletzki et al. ${ }^{54}$ have also stated that the strong dipole in $\mathrm{MgO}$ thin films deposited on $\operatorname{Ag}(111)$ leads to specific KPFM and topographical imaging conditions depending on the charge state of the tip. More recently even, Masago et al. ${ }^{55}$, using a theoretical approach based on DFT calculations, stressed the influence of the polarizability of atomic species in the apparent LCPD on Si surfaces and reach markedly similar conclusions as those of the present work.

Most of the aforementioned results indicate that polarization effects play a key role in the occurrence of SRE forces with two consequences in nc-AFM and KPFM: (i) They contribute to the total interaction force and are thus involved in the $\Delta f$ signal used to perform "topographical images" and (ii) despite about a few tens of pico-newtons ${ }^{43,56}$, they must be considered for interpreting the LCPD atomic scale contrast owing to their cross-coupled distance- and bias voltage-dependence. To a first approximation, SRE forces should be proportional to the square of the applied bias voltage $V_{b}$. Thus, in both KPFM and spectroscopy curves where the frequency detuning $\Delta f$ is recorded as a function of the applied bias voltage $V_{b}\left[\Delta f\left(V_{b}\right)\right.$ parabolas], it should be possible to detect the SRE contributions to the total interaction.

Besides imaging by means of KPFM, the aforementioned single point spectroscopy $\Delta f\left(V_{b}\right)$ parabolas and similar detection methods are more frequently used to detect local charges and charge transfer. Since the first experiments by Terris et al. ${ }^{57}$ where triboelectrification was measured with a sensitivity of a few charges only, single charges ${ }^{58-61}$, dipole moments ${ }^{62-64}$ as well as the charge state of individual point defects ${ }^{65}$ have been detected.

Due to their fundamental physical origin, SRE forces are ubiquitous. They are more and more under discussion since in recent years nc-AFM and KPFM are used to investigate 
single charges and local surface potentials on the nanometer length scale. In this work, we focus on the SRE interactions and ask two questions in regard to the imaging and the spectroscopy of charged species:

- can SRE forces alone be responsible for the atomic scale image contrast?

- is single charge detection on the nanometer scale possible?

These two questions have been raised by recent experimental results presented by Gross et $a l .{ }^{56}$. The authors present a set of experimental results combining single electron charge sensitivity and atomic lateral resolution the latter, however, in topography imaging and not in KPFM. The investigated system is a charged or neutral gold atom adsorbed on a thin dielectric layer $(\mathrm{NaCl})$ on a metal surface. The tip carries a foremost gold atom. Different charge states of the adatoms are clearly resolved in nc-AFM topography images and bias spectroscopy can be used to identify the charge state of the gold adatom. Note that for the reported experimental tip-sample distances $(>4.5 \AA)$, any interaction due to covalent binding can be excluded and only vdW and electrostatic forces have to be regarded. Therefore, these experiments are an ideal model case in order to study the role of dipolar forces in nc-AFM experiments.

In the present work, we clarify the role of SRE interaction in the topography imaging mechanism of single charges and we give the limits for lateral resolution in single charge detection. Thereto, we develop a relatively simple analytical model which mimics the experimental tip-sample geometry as presented by Gross et al. and we use it to calculate the experimental images (line profiles) as well as the spectroscopy curves. In addition to the well known vdW forces ${ }^{66}$ and the long-range electrostatic forces ${ }^{23,67}$ for a known tip geometry, our model includes a self-consistent description of the SRE interaction including electronic polarization effects. Our purpose is to show how SRE contribution can reproduce trends and orders of magnitude reported in the experiments by Gross et al. ${ }^{56}$. However, it is not our intention to fit their results since that would require a fully atomistic description involving a complex simulation process $^{14-18}$. 


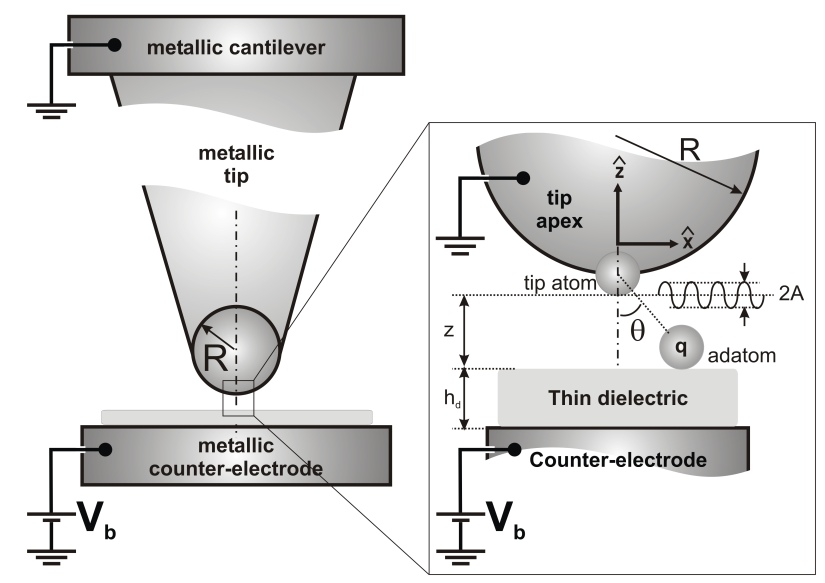

FIG. 1: Geometry of the problem. The tip consists of a macroscopic metallic body with a conical shape. The tip apex is described by a sphere with a radius $R$. The tip is grounded with regard to the counter-electrode on top of which lies a thin dielectric consisting of two mono-layers of an ionic film. The tip apex carries a neutral, but polarizable single atom. On top of the dielectric lies an adatom that may carry a single electron charge. The geometrical parameters of the problem are defined similarly to those in the work by Gross et al. ${ }^{56}$.

\section{ELECTROSTATIC MODEL}

\section{A. Framework}

The geometry of the problem is shown in Fig. 1. It accurately mimics the geometry of the problem as defined in the work by Gross et al. ${ }^{56}$. Our parameters are defined similarly as well. The tip consists of a metallic macroscopic body with a conical shape. The apical part of it is depicted by a sphere with a radius $R$. The apex carries a neutral single metallic atom in topmost position. The atom has an electronic polarizability $\alpha$. The tip is grounded with regard to the metallic counter-electrode located below a thin dielectric with a thickness $h_{d}$ and biased at a potential $V_{b}$. In Ref.[56], the dielectric is made of two mono-layers of $\mathrm{NaCl}$ deposited on a $\mathrm{Cu}(111)$ substrate. Thus, typically $h_{d} \simeq 3 \AA$. On top of the dielectric lies a metallic adatom that may carry a net single electron charge $q=\widetilde{q} \times e$, e being the elementary charge unit $\left(1.6 \times 10^{-19} \mathrm{C}\right)$. Hence, $\widetilde{q}= \pm 1$, or $\widetilde{q}=0$. It is assumed that the surface adatom has the same electronic polarizability $\alpha$ as the one present on the tip apex. 
Consistently with Ref.[56], the tip atom and the adatom are supposed to be Gold atoms. We therefore use the corresponding polarizability for that metal. In Ref.[68], pseudopotential calculations give an estimate for the dielectric susceptibility of Gold $\chi_{d}=\alpha \epsilon_{0}: 0.04 \times 10^{-39}<$ $\chi_{d}\left(\mathrm{~F} \cdot \mathrm{m}^{2}\right)<0.09 \times 10^{-39}$. Thus, we will consider in the following $\chi_{d}=0.0610^{-39} \mathrm{~F} \cdot \mathrm{m}^{2}$ and, hence, $\alpha=6.78 \times 10^{-30} \mathrm{~m}^{3}$. The instantaneous tip-surface separation is $z$, as defined in the figure. Even though the interfacial atoms are depicted with a spatial extension as in Ref.[56], they are treated as punctual objects in the model. Thus, it is important to notice that our definition of the tip-surface separation is similar to the one in the work by Gross et al.

When brought "close enough" to the surface, the tip becomes subject to forces, with essentially two origins ${ }^{69}$ : (i) Electrostatic forces comprise capacitive, Coulombic and what we henceforth refer to as SRE forces, so called dipolar forces, and (ii) vdW forces, among which are London dispersion forces if the interacting species do not carry permanent dipoles, as in the present situation. Dipolar forces, as we define them, have a microscopic origin and are connected to the ability of the atoms at the interface to get polarized owing to the dual influence of the biased electrodes and of the presence of the charge within the tip/counterelectrode capacitor. Thus, the tip atom and the surface adatom carrying the charge $q$ may acquire net dipolar moments, $\vec{p}_{t i p}$ and $\vec{p}_{a d}$, respectively. It is important to notice that these forces have a significantly different microscopic origin compared to vdW forces. This statement is justified in the following.

These forces have distinct decay lengthes, which permits to split their influence between the long-range $(\mathrm{LR})$ regime $(z>1 \mathrm{~nm})$ and the short-range $(\mathrm{SR})$ regime $(z<1 \mathrm{~nm})$. Therefore, the total force acting between the tip and the surface may be written as:

$$
\vec{F}_{t o t}=\vec{F}_{L R}+\vec{F}_{S R}=\left[\vec{F}_{L R}^{e l}+\vec{F}_{L R}^{v d W}\right]+\left[\vec{F}_{S R}^{l l}+\vec{F}_{S R}^{v d W}\right]
$$

The origins of LR- and SR-vdW forces are well-established. They are very briefly discussed in the following sections. At this stage, we focus on the electrostatic force, especially on the SR part of it, which has been under discussion in a few recent publications, as detailed in Sec. I.

In the present work, an expression of the SRE force regarding the system depicted in Fig. 1 is derived. The starting point of the approach is the expression of the electrostatic energy for the complete tip/vacuum/charge/dielectric layer/counter-electrode interface, $W^{e l}$ 
described in Eq. (2). The vertical component of the total electrostatic force acting on the tip $F_{t o t}^{e l}=F_{L R}^{e l}+F_{S R}^{e l}$ will then be derived as $F_{t o t}^{e l}=-\partial W^{e l} / \partial z$. The constitutive equation of the electrostatic energy for the system under investigation is

$$
W^{e l}=\left[-\frac{1}{2} C^{0} V_{b}^{2}+q \phi^{0}+\frac{1}{2} q \phi^{i m}\right]_{L R}+\left[-\frac{1}{2} \vec{p}_{t i p} \cdot \vec{E}_{t i p}^{e x t}-\frac{1}{2} \vec{p}_{a d} \cdot \vec{E}_{a d}^{e x t}\right]_{S R}
$$

The first bracket stands for the usual electrostatic energy of the tip/vacuum/dielectric layer/counter-electrode capacitor including the inner charge, as described for instance in Refs.[70,71]. In our problem, this term has a macroscopic origin since it depends on the geometry of the capacitor. Therefore, forces with a LR character will be associated with it. As said before, this term may be interpreted as the superposition between a purely capacitive force and Coulombic forces. $C^{0}$ depicts the capacitance of the former capacitor including neither the inner charge $q$ nor the microscopic dipoles $\vec{p}_{t i p}$ and $\vec{p}_{a d}$. This defines a void capacitor, the capacitance of which may be derived upon integration over its geometry. $\phi^{0}$ is the electrostatic potential which develops within the void capacitor at the location of the surface adatom, that is, the location of the charge $q . \phi^{i m}$ is the electrostatic potential induced by the set of image charges of $q$ within both metallic electrodes at its location.

Although usual in electrostatics ${ }^{72}$, the second bracket in Eq. (2) is less usual when dealing with nc-AFM. It depicts the electrostatic energy of microscopic dielectrics, here the tip atom and the charged adatom, when polarized by an external field. In the present case, on the microscopic level, we may expect that the adatom gets polarized by the electric field which develops between the tip and the counter-electrode referred to as $\vec{E}_{a d}^{e x t} . \vec{E}_{a d}^{e x t}$ results from the superposition of two components: the field within the void capacitor plus the field induced by the image charges of the charged adatom within the metallic electrodes. Thus, the adatom will acquire a dipolar moment $\vec{p}_{a d}$. Simultaneously, the tip atom gets polarized (dipolar moment $\vec{p}_{t i p}$ ) by the electric field $\vec{E}_{t i p}^{\text {ext }}$ that may be built in the same manner: the field of the void capacitor, the field induced by the image charges of the charged adatom within the electrodes, but also the Coulombic field developed by the charge itself. $\vec{E}_{a d}^{e x t}$ does not include the latter term since a charge can not influence itself.

Thus, the expressions of the microscopic dipoles $\vec{p}_{a d}$ and $\vec{p}_{t i p}$ and those of the external electric fields $\vec{E}_{a d}^{e x t}$ and $\vec{E}_{t i p}^{e x t}$ are to be derived. For that purpose, few assumptions are required. 


\section{B. Main assumptions}

It is assumed that the dipoles $\vec{p}_{\text {ad }}$ and $\vec{p}_{\text {tip }}$ have a microscopic origin because they are only connected to the electronic polarizability of the adatom and of the tip atom, $\alpha$. Hence, any depolarizing field is considered, as it should be the case with an extended dielectric. In order to keep the model as simple as possible, it is retstated that the tip atom and the surface adatom consist of the same material. Therefore they have the same polarizability $\alpha$, which is not strictly correct if the adatom is charged.

The influence of the thin dielectric layer within the capacitor is described by means of an effective dielectric permittivity $\epsilon_{\text {eff }}$, instead of $\epsilon_{0}$. This would be correct if one was dealing with a planar capacitor, which is false due to the shape of the tip apex. However, we show that dipolar forces have a SR character. Therefore, the strongest effects are expected to occur when the adatom is placed right below the tip atom at a distance of a few Angströms. Then, the "local capacitor" influencing the adatom is mainly localized within an almost planar area corresponding to the foremost part of the spherical apex ${ }^{73}$. This assumption is valid for $R \gg z$. The capacitance of a planar capacitor, the electrodes of which are at a distance of $h$ from each other, is $C=\epsilon_{0} / h$. When including a dielectric with a permittivity $\epsilon_{d}$ and a thickness $h_{d}$ between the electrodes, the capacitance becomes: $1 / C=$ $\left(h-h_{d}\right) / \epsilon_{0}+h_{d} / \epsilon_{d}$. Therefore: $C=\epsilon_{e f f} / h$, with $\epsilon_{e f f}=\epsilon_{0} \epsilon_{d} /\left[\epsilon_{d}\left(1-h_{d} / h\right)+\epsilon_{0} h_{d} / h\right]$. Considering parameters corresponding to the geometry of the problem: $h \simeq 8 \AA, h_{d} \simeq 3 \AA$, $\epsilon_{d}=5 \epsilon_{0}(\mathrm{NaCl}), \epsilon_{e f f} \simeq 1.4 \epsilon_{0}$. Thus, the influence of the discontinuous dielectric within the capacitor consists in replacing $\epsilon_{0}$ by an effective dielectric permittivity with a slightly larger value. The preceding assumption implies that the dielectric is treated as a continuous medium. Hence, no microscopic description of it is considered.

Following the preceding elements, the polarizing fields influencing the adatom and tip atom referred to as $\vec{E}_{a d}^{d i p}$ and $\vec{E}_{t i p}^{d i p}$, respectively (cf. Sec. IID), are calculated by assuming a planar geometry of the tip, which implies $R \gg z$ as well.

We do not address the influence of quantum effects in the problem and stick to a classical description. Hence, we attempt to describe neither the interaction between the adatom and the ionic layer nor the stability of the tip atom at the apex and the way its polarization might influence the local electronic density of the apex, thus modifying the resulting dipole, by screening effects for instance. In this approach, the interfacial atoms are somewhat frozen 
and the resulting interaction felt by the tip is measured at distances much larger than the tip atom/apex or adatom/ionic layer distances. These assumptions look reasonable in that the experimental results by Gross et al. are performed for tip-surface separations larger than $4 \AA$ and at $5 \mathrm{~K}$, which ensures the atomic stability of the tip apex and of the adatom over the surface. Nevertheless, from a classical point of view, the polarized tip atom creates an image dipole within the metallic apex, very close to the source dipole, with the same strength as it. Thus, because those dipoles are very close to each other, we assume that this ultimately produces an equivalent dipole built as their vectorial summation (cf. Appendix).

Because the problem is being treated in a classical manner, it is assumed that no wave function overlap between the electrons of the tip atom and those of the adatom takes place. In other words, so called "chemical SR interactions" are not taken into account. This implies that the tip-surface separation remains larger than 4-5 $\AA$, which is indeed the case in the work reported by Gross et al. At these distances, this also implies that the Madelung surface potential of the $\mathrm{NaCl}$ thin film is too weak to significantly modify the local electrostatic potential, owing to its exponential-like decay ${ }^{43,74}$. Thus, the influence of the Madelung potential on the resulting SRE force is not described. As a consequence, it must be noticed that the present description significantly differs from the models by Bocquet et al. ${ }^{43}$ and Giessibl $^{13}$ on a bulk alkali halide crystal, for which polarization effects uniquely stem from the Madelung surface potential.

For the sake of clarity, the way the analytical expressions are derived is not detailed in the text. We rather discuss the main issues deriving from the formal expressions. The details of the analytical method and the required calculations are extensively detailed in the Appendix.

\section{Long-range part of the total force}

The preceding elements give the following expression for the force acting between the tip and the surface in the LR regime:

$$
F_{L R}=F_{L R}^{v d W}+F_{L R}^{e l}=F_{L R}^{v d W}-\frac{\partial}{\partial z}\left[-\frac{1}{2} C^{0} V_{b}^{2}+q \phi^{0}+\frac{1}{2} q \phi^{i m}\right]
$$

$F_{L R}^{v d W}$ depicts the London dispersion force between the tip and the surface. It consists of two contributions. The first one is calculated in a cone+sphere/plane geometry, as described in 
Ref.[67] (Eq. 2.4). The cone is $1 \mu \mathrm{m}$-high with a $10^{\circ}$ open angle. The sphere has a radius $R$ (cf. Fig. 1), the value of which is specified in the following. The second vdW contribution is calculated between the surface adatom and the spherical apex of the tip ${ }^{66}$ :

$$
F_{a d \rightarrow t i p}^{v d W}(d)=-4 \pi C_{v d W} \rho R^{3}\left[\frac{1}{(d+2 R)^{4} d^{3}}+\frac{1}{(d+2 R)^{3} d^{4}}\right],
$$

with $d$ being the distance between the sphere' surface and the adatom center measured along the axis connecting it to the sphere's center. When assuming an interface consisting of similar materials, for example a gold adatom interacting with a gold tip, $C_{v d W}$ scales as $\alpha^{2}$ and is connected to the Hamaker constant of the tip-surface interface, $H$, according to $H=\pi^{2} \rho^{2} C_{v d W}$, where $\rho$ stands for the atomic density of the material. For gold, $\rho_{A u}=$ $6 \times 10^{28}$ at. $\mathrm{m}^{-3}$ and for metals, it is known ${ }^{66}$ that $H=1-5 \times 10^{-19} \mathrm{~J}$. In the following, we take $H_{A u}=3 \times 10^{-19} \mathrm{~J}$. Therefore $C_{v d W}=0.8 \times 10^{-77}{\mathrm{~J} . \mathrm{m}^{6}}$.

The capacitive force involving the capacitance of the void capacitor $C^{0}$ was derived from Ref.[67] as well (Eq. (2.3)). The expressions of the potentials $\phi^{0}$ and $\phi^{i m}$ are derived by neglecting the influence of the conical part of the tip apex. They are calculated by the process of image charges of the charge $q$ between the counter-electrode and the sphere standing for the apex of the tip, similarly to the method extensively detailed in Refs.[70-72].

\section{Short-range part of the total force}

The SR part of the total force is:

$$
F_{S R}=F_{S R}^{v d W}+F_{S R}^{e l}=F_{S R}^{v d W}-\frac{\partial}{\partial z}\left[-\frac{1}{2} \vec{p}_{t i p} \cdot \vec{E}_{t i p}^{e x t}-\frac{1}{2} \vec{p}_{a d} \cdot \vec{E}_{a d}^{e x t}\right]
$$

$F_{S R}^{v d W}$ depicts the SR London dispersion force between the interfacial atoms, that is the tip atom and the adatom. The latter component occurs whether the adatom is charged or not. Most of the time, when the interacting atoms may be assumed as punctual objects, the interaction is described by a Lennard-Jones potential scaling as $r^{-6}$. Hence: $F_{S R}^{v d W}=-6 C_{v d W} / r^{7}$. According to the estimated value for $C_{v d W}$, one has used: $F_{S R}^{v d W}(n N) \simeq-500 / r^{7}(\AA)$.

We now focus on the description of the dipolar force. The expressions of the microscopic dipoles $\vec{p}_{t i p}$ and $\vec{p}_{a d}$ and of the external fields $\vec{E}_{t i p}^{e x t}$ and $\vec{E}_{a d}^{e x t}$ are to be found. However, the microscopic dipoles arise from the local electric field felt by the tip atom or by the adatom ${ }^{75}$ 
including, in addition to the external field, the mutual influence of the dipoles on each other, thus ensuring the self-consistency of the model. Therefore,

$$
\vec{p}_{t i p, a d}=\epsilon_{0} \alpha \vec{E}_{t i p, a d}^{l o c}
$$

with

$$
\vec{E}_{t i p, a d}^{l o c}=\vec{E}_{t i p, a d}^{e x t}+\vec{E}_{t i p, a d}^{d i p}
$$

$\vec{E}_{t i p}^{d i p}\left(\vec{E}_{a d}^{d i p}\right)$ is the dipolar field induced by the polarized adatom (the polarized tip atom), its set of image dipoles, but also by the image dipoles of the polarized tip atom (the polarized adatom), influencing the tip (the adatom). The preceding equation states that the polarizabilities of the tip atom and of the adatom are similar, consistent with our assumption.

Physically, the external fields $\vec{E}_{t i p, a d}^{e x t}$ are both proportional to $V_{b}$ and $q$ [visible in Eqs. (A.13), (A.14), (A.15) and (A.19), (A.20) in the Appendix). Besides, the self-consistency of the approach implies that $\vec{p}_{t i p, a d}$ stem from polarization effects, that is from a field, or a potential, able to trigger them. In turn, the dipolar fields $\vec{E}_{t i p, a d}^{d i p} \neq \overrightarrow{0}$ if and only if $\vec{p}_{\text {tip,ad }} \neq \overrightarrow{0}$. This requires either $V_{b} \neq 0$, or $\widetilde{q} \neq 0$ (in this case, the Coulombic potential created by the charge is $\neq 0$ ). For instance, the case $V_{b} \neq 0$ and $\widetilde{q}=0$ gives rise to dipolar forces because a neutral adatom may be polarized. Conversely, the peculiar case $V_{b}=0$ and $\widetilde{q}=0$, implies $\vec{E}_{t i p, a d}^{\text {ext }}=\overrightarrow{0}$ and thus $\vec{E}_{t i p, a d}^{\text {dip }}=\overrightarrow{0}$ because $\vec{p}_{t i p, a d}=\overrightarrow{0}$. Then no dipolar forces occur, and the resulting SR force is only driven by the SR-vdW interaction.

The formal expressions of the external and local electric fields are given in appendix. The lateral ( $x$ axis) and vertical ( $z$ axis) expressions of $\vec{p}_{a d}$ and $\vec{p}_{t i p}$ are derived by solving a set of linear equations [cf. Appendix, Eqs. (A.17) and (A.22)]. Finally, the vertical component of the SRE force, $F_{S R}^{e l}$, may be derived by differentiation with respect to $z$ [Eq. (5)], and thus the expression of the total force may be obtained. The problem thus rationalized relies on two main parameters, namely, the polarizability of the interfacial atoms $\alpha$ and the Hamaker constant of the interface $H$.

The microscopic origin of the dipolar forces described here differs significantly from the origin of vdW forces between atoms or molecules, among which are London dispersion forces (generic term $F_{v d W}$ in the model), but also Debye or Keesom forces (not described as they are irrelevant with the present setup). London dispersion forces stem from the net dipolar 
interaction resulting from the instantaneous electronic polarization of the interacting atoms. They occur whether the interacting atoms are charged or not and whether a bias voltage is applied or not. Debye forces stem from the interaction between an atom or a molecule carrying a permanent dipole and an induced dipole. Keesom forces stem from the interaction between two permanent dipoles subject to random orientation due to thermal motion. Here, dipolar forces do rely on the electric biasing of the electrodes and on the presence of net charges within the tip-surface interface because the induced fields polarize the interfacial atoms that subsequently self-influence themselves. Furthermore, they do not require the atoms to carry permanent dipoles and are not sensitive to thermal effects because the atomic positions are considered to be frozen and the electronic polarization is independent of the temperature.

\section{ANALYTICAL RESULTS AND COMPARISON WITH EXPERIMENTAL RESULTS}

A. SR-electrostatic versus SR-vdW forces: strength, lateral resolution and distance dependence

In Fig. 2(a), the SR force including vdW and dipolar contributions, $F_{S R}=F_{S R}^{v d W}+F_{S R}^{e l}$, is drawn as a function of the lateral position of the tip depicted by its angle $\theta$ with respect to the charged adatom $(\theta=0$, cf. Fig. 1$)$. The force curves have been computed with $V_{b}=0$ for two tip-surface separations, $z=4.8$ and $5.1 \AA$ and above $\widetilde{q}=-1$ (charged adatom) and $\widetilde{q}=0$ (neutral adatom). The unchanged parameters are: polarizability for gold $\alpha=6.78 \AA^{3}$, dielectric thickness $h_{d}=3.0 \AA$ (two NaCl layers), and $\epsilon_{e f f}=1.4 \epsilon_{0}$. These force profiles are to be compared to the experimental ones shown in Fig. 2(B) of Ref.[56] measured on $\mathrm{Au}^{-}$ and $\mathrm{Au}^{0}$, for which the LR background force has been subtracted. In addition, we have assumed the two atoms are far enough apart $(\simeq 27 \AA$ in the experimental work) to avoid a potential cross-influence in the resulting force measured on top of each of them.

Let us consider the case $\widetilde{q}=0$ first [neutral adatom, green curves in Fig. 2(a)]. No dipolar force occurs because $V_{b}=0$ and $\widetilde{q}=0$. The resulting interaction is only driven by the SR-vdW force, as stated in Sec. II D. We stick to the experimental values of the force measured at $z=4.8 \AA$ in Ref.[56] [yellow curve in Fig. 2(B) of the reference]. The numerical 

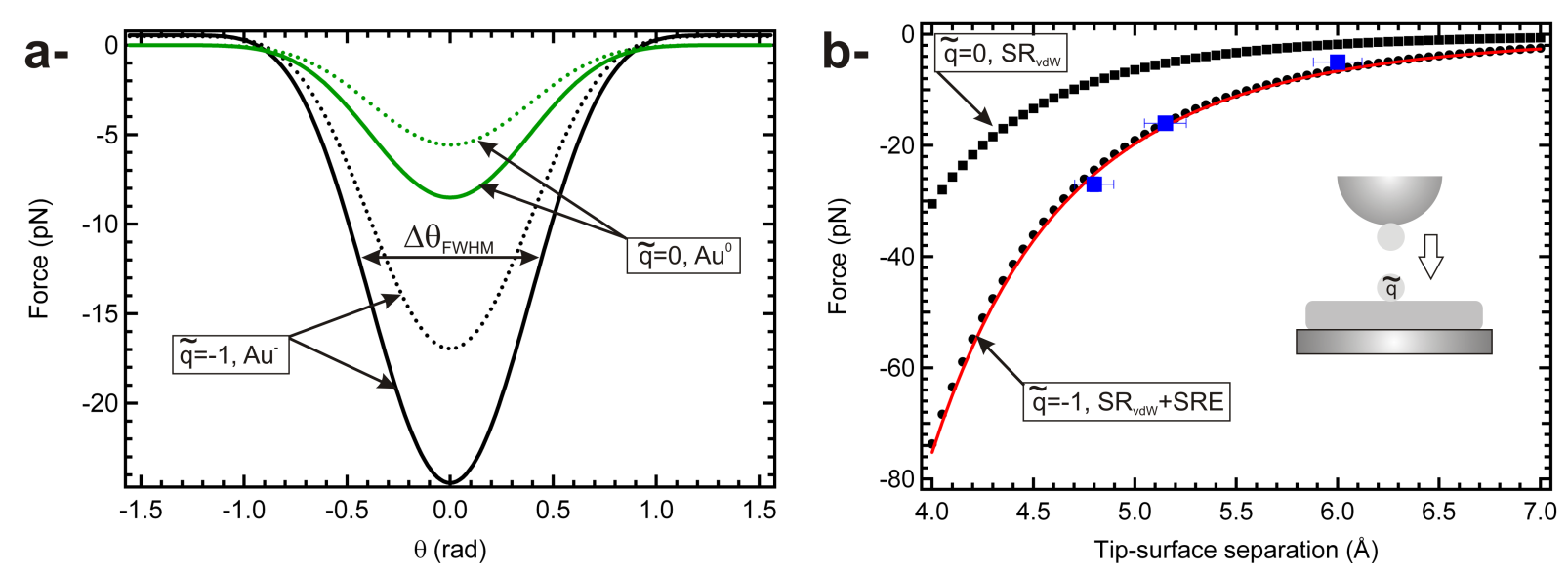

FIG. 2: (Color online). (a) Tip-adatom SR force including SR-vdW and dipolar contributions as a function of the angular position of the tip with respect to the adatom located in $\theta=0$. The force profiles have been computed with $V_{b}=0$ and $z=4.8 \AA$ (continuous curves) and $5.1 \AA$ (dotted curves), ont top of $\widetilde{q}=-1$ (black curves) and $\widetilde{q}=0$ (green curves). The full width at half-maximum for the black curve $(\widetilde{q}=-1, z=4.8 \AA)$ is $\Delta \theta= \pm 0.44 \mathrm{rad}$. The comparison between the numerical results and the experimental curves from Gross et al. ${ }^{56}$ is good (cf. text). (b) Distance dependence of the tip-adatom SR force computed with $V_{b}=0$ on top of $\widetilde{q}=-1$ (black dots) and on top of $\widetilde{q}=0$ (squares). The latter curve merely consists of the SR-vdW force component $\left(\propto z^{-7}\right)$. The fit of the curve measured on top of $\widetilde{q}=-1$ (continuous red curve) has been obtained with a $z^{-6}$ function. The experimental values of the force derived from Gross et al. are depicted with blue squares. The horizontal error bars depict a possible uncertainty in the $z$ position of the tip of $2 \%$ only. The discrepancy between SRE and SR-vdW forces is well visible.

estimate of the force gives $-8.5 \mathrm{pN}$ whereas the reported experimental force rather is $-14 \mathrm{pN}$. This discrepancy may be reduced if one considers a sphere-sphere vdW interaction instead of vdW interaction between atoms treated as punctual objects. The vdW interaction force between spheres may be derived from Eq. (1) in Ref.[76]. With two gold atoms (144 pm

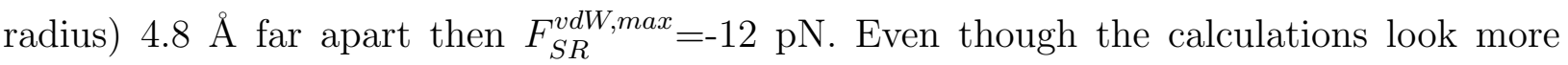
accurate, we keep on using the vdW interaction between punctual objects in the following in order to: (i) be consistent with the punctual-like description of the charged adatom for the electrostatic interaction and (ii) not bring further uncertainty in the calculations, as the value of the $\mathrm{Au}^{-}$radius was not found.

When now considering the curve computed on top of $\widetilde{q}=-1$ at $z=4.8 \AA$ [continuous 
black curve in Fig. 2(a)], the numerical estimate gives $-24.5 \mathrm{pN}$ and the experimental value of the force reaches $-27 \mathrm{pN}$. At $z=5.1 \AA$ (dotted black curve), the force has decayed down to $-16.9 \mathrm{pN}$, corresponding to a $\mathrm{max} / \mathrm{min}$ ratio of 1.45 within $\Delta z=30 \mathrm{pm}$ only. Therefore in the present situation, SR-vdW forces are not able to account for the experimental variations observed on top of $\mathrm{Au}^{-}$, which states that SRE forces weight significantly in the total force upon the polarizability and the charge state of the interfacial atoms.

In Fig. 2(a), it is restated that the calculations are performed with (i) both $\widetilde{q}=0$ or $\widetilde{q}=-1$ adatoms lying on the dielectric' surface and hence located at the same separation with regard to the tip atom when placed above them and (ii) with the same polarizability $\alpha$ for them. Thus, this allows us to strictly compare SRE and SR-vdW forces, while getting rid of the topographical influence and of the chemical nature of the underlying adatoms, respectively. We are aware that these assumptions are problematic. Indeed, regarding issue (i), it is known that, when adsorbed on a $\mathrm{NaCl}$ thin film, $\mathrm{Au}^{-}$embeds within the surface by about $0.4 \AA$, whereas the neutral gold atom remains at the surface ${ }^{77}$. Regarding issue (ii), it is obviously expected $\alpha\left(\mathrm{Au}^{-}\right)>\alpha\left(\mathrm{Au}^{0}\right)$. Nevertheless, a rather good agreement is observed between the calculations and the experiments. We think this stems from the two following effects that actually balance each other: the underestimation of the tip- $\mathrm{Au}^{-}$separation, which implies an overestimation of the force [as it decays quickly with $z$; cf., Figs. 2(a) and 2(b)], and an underestimation of the force on top of $\mathrm{Au}^{-}$, as all the SR forces increase with $\alpha$.

The force profile as a function of the lateral position of the adatom allows us to draw conclusions regarding the lateral resolution of neutral and charged adatoms in the experimental images. This requires first to define a criterion stating unambiguously what the lateral resolution is. We stick to the following criterion: two objects separated by a distance $d$ over the surface are assumed as being resolved by the tip if the full width at half maximum (FWHM) of their respective force profile $\left[\Delta x_{F W H M}^{(1)}\right.$ and $\Delta x_{F W H M}^{(2)}$, respectively] do not overlap, that is $d>\left(\Delta x_{F W H M}^{(1)}+\Delta x_{F W H M}^{(2)}\right) / 2$. In the range 4.8$5.1 \AA$, the calculated FWHM force profile on the negatively charged adatom or on the neutral one does not vary much: $\theta_{F W H M} \simeq \pm 0.44 \mathrm{rad}$. Therefore, at $z=4.8 \AA$, $\Delta x_{F W H M}\left(\mathrm{Au}^{-}\right)=\Delta x_{F W H M}\left(\mathrm{Au}^{0}\right)=2 z \times \tan \left(\theta_{F W H M}\right)=4.5 \AA$. The experimental values are: $\Delta x_{F W H M}\left(\mathrm{Au}^{-}\right) \simeq 5 \AA$ and $\Delta x_{F W H M}\left(\mathrm{Au}^{0}\right) \simeq 7.5 \AA$. If the comparison to the experiments is good in the case of $\mathrm{Au}^{-}$, which testifies to the importance of the SRE forces contribution, 
there is a significant discrepancy with $\mathrm{Au}^{0}$. At this stage, this is not yet understood. But, regarding the spatial extension of the force profile on a single electron charge, the "apparent size" of a charged adatom is a few Angströms only. This is a somewhat unexpected result in that a naive view of the problem might have expected a usual Coulombic $z^{-1}$ dependence, thus preventing any high-resolution imaging of these objects from being performed.

In Fig. 2(b), the distance dependence of the total SR force on top of an adatom with $\widetilde{q}=-1$ is reported (black dots) and compared to the experimental data from Gross et al. (blued squares). These have been derived for few tip-surface separations given in the reference [Fig. 2(B); $z=4.8,5.15$, and $6.0 \AA$ ]. The agreement between the experimental and the numerical data is still good, within however deviations due to the limitations of the model and to the experimental error bars. For instance, the $z$ error bars shown in Fig. 2(b) correspond to $\pm 2 \%$ only. The best fitting function for the force was found to be $z^{-6}$ (continuous red line). For comparison, we have as well drawn the SR-vdW curve (i.e. on top of $\mathrm{Au}^{0}$ ) scaling in $z^{-7}$ (black squares), the behavior of which is significantly different. This trend is fully consistent with the one shown in Fig. (S1B) of the supporting online material of Ref.[56]. Thus, SRE forces decay almost as fast as SR-vdW, but their strength may prevail on the SR-vdW contribution upon the polarizability and/or the charge state of the interfacial atoms.

\section{B. SR- versus LR-electrostatic forces: influence on the charge state characteriza-} tion

A major aspect of the work by Gross et al. deals with the detection of single electron charges by means of the measurement of the LCPD. The authors showed that the LCPD might selectively be shifted upon the sign of the charge of the underlying adatom (positive, neutral or negative). We hereafter detail the bias voltage dependence of the total force derived from the model upon the sign of the adatom and discuss how the LR-electrostatic component may influence the detected LCPD.

In order to stick to the experimental results of Gross et al. shown in Fig. (3A) of the reference, the spectroscopic curves are reported in terms of frequency shift $(\Delta f)$ as a function of the dc bias. For that purpose, the expression of $\Delta f$ is derived from the force $F$ according to a simplified expression, only valid when using small oscillation amplitudes ${ }^{78,79}$ as this is 

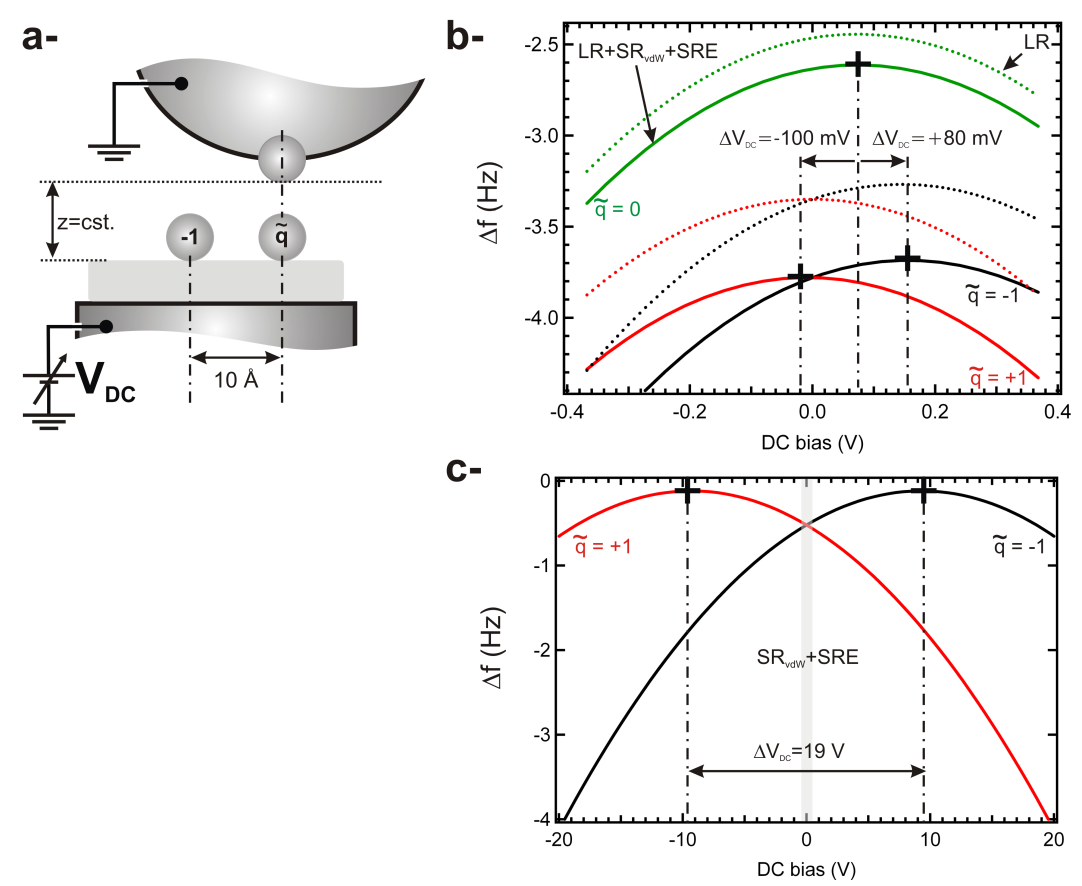

FIG. 3: (Color online). (a) Geometry used to compute the spectroscopic curves shown in panel (b). (b) $\Delta f$ vs. dc bias spectroscopic curves computed for $\widetilde{q}=+1$ (red), $\widetilde{q}=0$ (green), and $\widetilde{q}=-1$ (black) at $z=5.8 \AA$. The tip apex's radius has been set equal to $R=50 \AA$. The numerical curves are found to be in good agreement with the experimental results measured on top of $\mathrm{Au}^{0}$ and $\mathrm{Au}^{-}$. Note also that the way the LCPD is shifted (larger values with negatively charged adatoms and vice-versa) is consistent with the experimental findings. To assess the influence of SRE forces, the curves have been plotted with (continuous lines) and without (dotted lines) the SR components. SRE forces do not contribute significantly to shift the LCPD upon the charge state of the adatom (similar positions of the parabolas' maxima), but rather shift the total force. Therefore, with the present setup, the LR-electrostatic interaction is solely responsible for the observed LCPD shift $(\Delta V=+80$ or $-100 \mathrm{mV})$. (c) $\Delta f\left(V_{b}\right)$ curves computed above $\widetilde{q}=-1$ (black curve) and $\widetilde{q}=+1$ (red curve) upon similar conditions as (b), except that the LR components are set equal to 0 . The shaded area depicts the range of bias shown in (b). Over that range, the variation of the SRE-connected $\Delta f$ is very small, despite a strong splitting between parabolas' maxima.

the case in Gross' experiments:

$$
\Delta f=-\frac{f_{0}}{2 k} \frac{\partial F}{\partial z}
$$


In Fig. 3, $\Delta f$ vs. $V_{D C}$ curves are reported. They have been computed on top of an adatom with $\widetilde{q}=+1, \widetilde{q}=0$ or $\widetilde{q}=-1$. We have used the experimental parameters given in Ref.[56]: $k=1800 \mathrm{~N} / \mathrm{m}$ and $f_{0}=23000 \mathrm{~Hz}$. The curves are computed at constant height, $z=5.8 \AA$ with a tip radius $R=50 \AA$ and an additional adatom with a charge state $\widetilde{q}=-1$, located $10 \AA$ aside the one on top of which the spectroscopic measurement is performed [cf. Fig. 3(a)]. This is consistent with the geometry of the spectrometric measurements performed by Gross shown in Figs. 3(A), (B) and (C). Note that the spectroscopic curve computed on top of $\widetilde{q}=+1$ is for predictive purpose as no equivalent curve is reported in the experimental work. Let us focus on the curves corresponding to the total force first [continuous lines labeled as $\mathrm{LR}+\mathrm{SR}_{\mathrm{vdW}}+\mathrm{SRE}$ in Fig. 3(b)]. The qualitative behavior between numerical and experimental results is good and similar trends are observed. First, over a similar range of voltage $( \pm 400 \mathrm{mV})$, the numerical values of $\Delta f$ range from -2.4 down to $-4.4 \mathrm{~Hz}$, while the experimental range is $-2.4 . .-3.3 \mathrm{~Hz}$. We assign this discrepancy to our assumption of a sphere-plane capacitor for the calculation of the potential $\phi^{0}$ and $\phi^{i m}$ in the LR-electrostatic interaction. Second, negatively charged adatoms have a larger LCPD than neutral and positively (not shown in the experimental results) charged ones. The gap voltage between $\widetilde{q}=0$ and $\widetilde{q}=-1$ is $\Delta V=+80 \mathrm{mV}$, to be compared to the experimental value: $\Delta V \simeq+30 \mathrm{mV}$. Third, a larger attractive force occurs on top of $\widetilde{q}= \pm 1$ (more negative shift) than on $\widetilde{q}=0$, consistent with the experimental findings and with the results of the preceding section. Fourth, the maxima of the numerical curve measured on top of $\widetilde{q}=0$ is not centered around 0 , but rather around $V_{D C}=+80 \mathrm{mV}$, to be compared to the $180 \mathrm{mV}$-experimental value. This effect stems from the electrostatic influence of the additional adatom $\widetilde{q}=-1$ located nearby in the capacitor, which contributes to the positive shift of the LCPD, consistent with what is observed on top of $\widetilde{q}=-1$. This is also the effect that makes the LCPD calculated on top of $\widetilde{q}=+1$ almost zero since the total charge within the capacitor is then zero.

We now discuss the relative weight between LR and SR forces in $\Delta f\left(V_{b}\right)$ spectroscopic curves. To first order, dipolar forces scale as $V_{b}^{2}$ (cf. Appendix), but the bias-dependence of the total force as well stems from the LR-electrostatic part of the interaction. In order to understand what force component is the most significant in the bias dependence, spectroscopic curves computed without SR forces, that is only with the LR components, are drawn in the figure [dotted curves labeled as LR in Fig. 3(b)]. The parabolas are not bias-shifted, 
but $\Delta f$-shifted (less negative values), upon removal of SRE forces. Indeed, when investigating the bias dependence of the mere SRE forces above $\widetilde{q}=-1$ and $\widetilde{q}=+1$ upon similar conditions [geometry, height; cf. Fig. 3(c)], it is found that over the range $\pm 400 \mathrm{mV}$ (shaded area in the figure), the curvature of the parabolas is very weak, corresponding to $\Delta f$ changes of about $0.1 \mathrm{~Hz}$. However, a large splitting between parabolas' maxima $\left(\Delta V_{D C}=19 \mathrm{~V}\right)$ is observed, which states that SRE forces are extremely sensitive to the charge state of the adatom as soon as LR-electrostatic forces are not present in the tip/dielectric/counterelectrode capacitor. Conversely, when these are present, the spectroscopic curves become weakly influenced by SRE components, which fully agrees with the pioneer work by Terris et $a l .{ }^{57}$. In this case, any attempt to accurately fit $\Delta f\left(V_{b}\right)$ experimental data requires a precise calculation of the electric field within the capacitor ${ }^{40,80,81}$, and therefore a precise knowledge of its geometry.

\section{SR- versus LR-electrostatic forces: influence on the lateral resolution}

We here address the issue of the lateral resolution as a function of SR- and LR-contributions. The conditions to achieve high-resolution imaging rely on SR interactions, including dipolar as well as SR-vdW forces, but also on the tip sharpness, that is the size of the tip apex.

For that purpose, we mimic the experimental situation shown in Fig. 2(A) of the work by Gross et al. A negatively charged adatom $(\widetilde{q}=-1)$ lying on the thin dielectric is placed at a distance $d=25 \AA$ from a neutral adatom $(\widetilde{q}=0)$. In Fig. $4, \Delta f$ profiles are reported as a function of the tip size and force components. These are derived from the corresponding force profiles calculated at $z=4.8 \AA$ with $V_{b}=0$ according to the same method as before. For each case reported in the figure, we have plotted the total force including LR and SR components (red curves) and solely the LR component (including vdW and electrostatics, black curves). The LR force is calculated for tip apexes corresponding to: (i) a hemispherical apex with $\mathrm{R}=20 \AA$ [Fig. 4(a), $R \simeq d$ ], (ii) same as (i) with $\mathrm{R}=45 \AA$ [Fig. 4(b), $R>d$ ], (iii) same as (i) with $\mathrm{R}=200 \AA$ [Fig. $4(\mathrm{c}), R \gg d$ ], and (iv) same as (i) with $R=1000 \AA$ [Fig. $4(\mathrm{~d})]$.

Let us first discuss the influence of the LR interaction on the calculated $\Delta f$ profiles. As expected, the LR interaction increases with the size of the macroscopic tip and produces a 

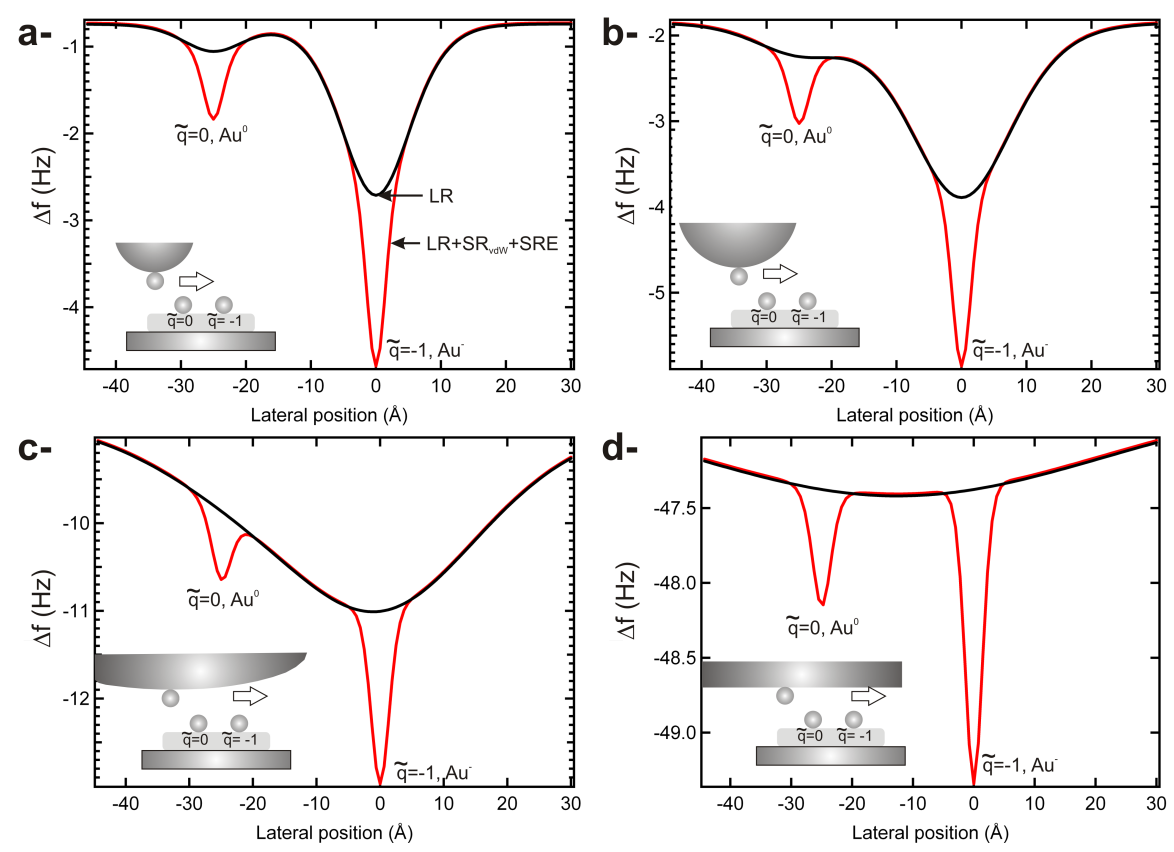

FIG. 4: (color online). $\Delta f$ profiles computed from the corresponding force profiles calculated at $z=4.8 \AA$ with $V_{b}=0$. The charged adatom $(\widetilde{q}=-1)$ is placed in $x=0$ and the neutral adatom is placed at a distance $d$ corresponding to $x=-25 \AA$. Cases a-, b-, c- and d- correspond to a tip with hemispherical apex $\mathrm{R}=20 \AA(R \simeq d), \mathrm{R}=45 \AA(R>d), \mathrm{R}=200 \AA(R \gg d)$ and a flat apex with an area equivalent to a hemispherical apex of radius $R=1000 \AA$, respectively. For each case, the total force (red curves) or the total force minus the SR components (black curves) has been used to derive the $\Delta f$ profile.

$\Delta f$ offset ranging from $-1 \mathrm{~Hz}$ in Fig. 4(a) up to $-47 \mathrm{~Hz}$ in Fig. 4(d). At the distances used in the experimental setup, it is the LR electrostatic interaction which dominates over the LR-vdW one for the sharpest tip. This can be seen in Fig. 4(a) where the different contrast of the black curves above $\mathrm{Au}^{0}$ and $\mathrm{Au}^{-}$is caused by the LR electrostatic interaction only. In the present case of zero bias $\left(V_{b}=0\right.$, and hence $\left.\phi^{0}=0\right)$ and a tip radius $R$ which is much larger than the distance $z(R>5 z)$, the LR electrostatic interaction only depends on the charge and the induced potentials $\phi^{i m}$ [see Eq. (2)] and preserves a more or less constant strength $(\simeq 2 \mathrm{~Hz})$ for all tip sizes. It is always centered around the charged adatom, however, its lateral confinement is decreasing with increasing tip size. Other than the LR electrostatic forces which are constant in the present setup, the LR-vdW forces scale with the volume of the tip. This is why for the flat tip calculated in Fig. 4(d) it is the LR-vdW which 
produces the largest part of the interaction. The LR-vdW is not sensitive to the charge; consequently, the maximum contrast is now centered between the two adatoms and no longer above the charged one. Please note that the LR-vdW part contains two contributions. One describes the interaction between the adatoms and the macroscopic tip; the other describes the interaction between the surface and the macroscopic tip. It is the first one which causes the parabolic shape of the black curve in Fig. 4(d), the latter one just adds a constant offset to the calculated $\Delta f$.

All the previously described LR interactions produce a more or less structured background in the $\Delta f$ profiles due to a convolution with the macroscopic tip shape. As soon as SRE forces are present, the convolution with the tip apex does not prohibit resolving charged adatoms. The main reason is that SRE forces are able to spatially and selectively pin the force on top of each adatom because they prevail on SR-vdW forces, as discussed in subsection II D. However, the lateral resolution depends on the exact shape of the apex. Surprisingly, a tip with an apex the size of which is significantly larger than the separation between the species to be resolved, provides the maximum of lateral resolution. Indeed, the influence of LR components is then averaged over the area of the tip, whereas the spatial distribution of the SR dipolar and vdW forces remains unchanged as they are not apexsize dependent. Conversely, this tip will provide no sensitivity to the charge state of the adatoms because the LR-electrostatic contribution will weight too much in the resulting bias dependence of the total force, as described in subsection III B. A very sharp tip [case (i)] will provide high resolution as well, as the one used by Gross et al. to perform their experiments, $R \simeq 5-10 \AA$. Between these extreme cases, the resolution will depend on the size of the tip apex, as discussed, for instance, in Ref.[82].

\section{SUMMARY AND CONCLUSION}

We present a model which explains in detail the nc-AFM experiments reported by Gross et $a l .{ }^{56}$. In these experiments, nc-AFM is used first to image the topography of charged and neutral gold atoms adsorbed on a thin dielectric layer deposited on a metal substrate. Second, frequency shift $\Delta f$ as a function of bias voltage $V_{b}$ curves are used to identify the charge state of these adatoms.

Our model mimics the experimental tip-sample geometry and accounts for both LR- and 
SR-vdW, as well as electrostatic forces. The geometry consists of a metallic tip and a metal substrate covered by two monolayers $(3 \AA)$ of a dielectric film. On both, tip and surface, there is a (polarizable) gold atom adsorbed. The SR chemical binding forces and displacements of atoms are not included since in the experiments, the closest tip-sample distance is larger than $4.5 \AA$. All material constants in our model are chosen according to values given in the literature. The model is based on the calculation of the electrostatic energy for the complete geometrical system. In addition to the well known terms of LR- and SR-vdW, as well as the LR-electrostatic terms, our model includes a less common term of SR-electrostatic (SRE) forces stemming from the self-consistent description of electronic polarization at the tipsurface interface including the contribution of image charges and image dipoles. These SRE interactions, so called dipolar forces, have a microscopic origin due to the polarizable atoms adsorbed on the tip front end and on the surface. The polarization of these atoms is due to the biased electrodes (metallic tip and counter-electrode), as well as to charges within the tip/counter-electrode capacitor.

The previously described SRE interactions are weak in comparison to SR binding forces. The experiments by Gross et al. were performed at distances larger than $4.5 \AA$ and therefore serve as model case experiments since SR binding forces are negligible. In this model case, it is found that the interaction responsible for the atomic scale topographical contrast in ncAFM is not determined by SR-vdW, but rather dominated by SRE interactions. However, for the identification of the charge state of the adsorbed atoms by means of $\Delta f\left(V_{b}\right)$ spectroscopy, our calculations clearly point out that the experimentally observed variation of the LCPD is dominated by the LR-electrostatic interactions. Thus, a different interaction allows for the discrimination of charged adatoms in topography imaging and in $\Delta f\left(V_{b}\right)$ spectroscopy, respectively.

These findings allow us to draw conclusions regarding experimental strategies to improve the spatial resolution on isolated charged species and/or high spectroscopic sensitivity to their charge state. In particular, the proposed experimental geometries might be the key to success in order to investigate surfaces that will be influenced or even destroyed when the tip apex approaches within the range of atomic binding distances.

- The topographical appearance of charged adatoms adsorbed on a dielectric relies on SRE forces, their high-resolution imaging requires a polarizable tip apex, but not mandatorily ultra-sharp. The latter issue however, would obviously facilitate the high- 
resolution. Polarizable tips might be achieved by using a properly-grafted polarizable molecule at the tip apex, or by using a tip coating consisting of a high-K material, for instance.

- The charge sensitivity in either the spectroscopic signature or KPFM measurements is hindered by LR-capacitive forces. In order to increase sensitivity, SRE contribution should be predominant. Thereto, two suggestions can be made. The first one is to increase the SRE contribution by using both, a polarizable tip apex as well as a sharp tip in order to increase the local electric field. The second concept is to make the tip/counter-electrode capacitance as small as possible. For that purpose, using a bulk insulator instead of a thin layer might be advantageous.

Choosing such experimental conditions would allow, for example, the detection of the local charge distribution within a single dipolar molecule.

\section{Acknowledgments}

The authors would like to thank Leo Gross and Gerhard Meyer for valuable comments and acknowledge financial support of the Centre National de la Recherche Scientifique, of the Région Provence Alpes Côtes-dAzur, as well as of the Agence Nationale de la Recherche with the PNANO project MolSiC (ANR-08-P058-36).

\section{APPENDIX}

The geometry of the parameters defined hereafter is detailed in Fig. 5.

\section{A. Summary of the analytical method and main assumptions}

The vertical component of the SR dipolar force to calculate derives from the classical equation:

$$
F_{S R}^{e l}=-\frac{\partial}{\partial z}\left[-\frac{1}{2} \vec{p}_{t i p} \cdot \vec{E}_{t i p}^{e x t}-\frac{1}{2} \vec{p}_{a d} \cdot \vec{E}_{a d}^{e x t}\right]
$$




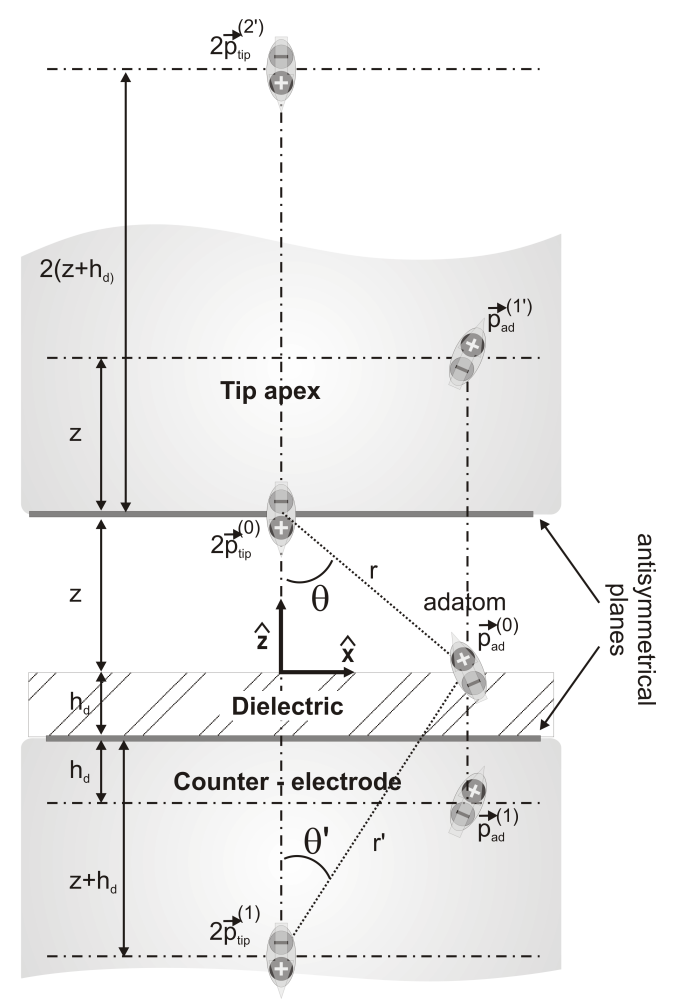

FIG. 5: Geometry of the parameters defined in the model and description of the image dipole process for the calculation of the dipolar fields, $\mathbf{E}_{\text {ad }}^{\mathbf{d i p}}$ and $\mathbf{E}_{\text {tip }}^{\mathbf{d i p}}$. For that calculation, the metallic tip apex is assumed as being planar (cf. text). Owing to the image process, this plane as well as the counter-electrode plane are antisymmetrical. Indexes (0) refer to the real dipoles. The set of image dipoles within the tip apex are numbered with a prime $\left(^{\prime}\right)$. For the calculation of the dipolar fields, we have solely used the set of images dipoles depicted in the figure (cf. text).

where $\vec{E}_{t i p}^{\text {ext }}$ and $\vec{E}_{a d}^{\text {ext }}$ are the external electric fields influencing the foremost tip atom and the surface adatom, respectively. These are produced by the biased void capacitor, the charged adatom and its set of image charges within the metallic electrodes.

In addition to the latter external fields, the self-consistency of the problem is ensured by the assumption that the adatom is as well polarized by the dipolar field of the tip atom and vice versa:

$$
\vec{p}_{t i p, a d}=\epsilon_{0} \alpha \vec{E}_{t i p, a d}^{l o c}
$$

with: 


$$
\vec{E}_{t i p, a d}^{l o c}=\vec{E}_{t i p, a d}^{e x t}+\vec{E}_{t i p, a d}^{d i p}
$$

$\vec{E}_{t i p}^{d i p}$ is the dipolar field polarizing the foremost tip atom. It originates from the following:

- the polarized adatom

- the set of image dipoles of the polarized adatom within the metallic electrodes

- the set of image dipoles of the polarized tip atom within the metallic electrodes

$\vec{E}_{a d}^{d i p}$ is the dipolar field polarizing the surface adatom. It originates from:

- the polarized tip atom

- the set of image dipoles of the polarized adatom within the metallic electrodes

- the set of image dipoles of the polarized tip atom within the metallic electrodes

The dipolar field in a point $M$ of the space produced by an isolated dipole $\vec{p}$ located in a point $O$, such that $\overrightarrow{O M}=r \widehat{e}_{r}$, is:

$$
\vec{E}^{d i p}(M)=\frac{1}{4 \pi \epsilon_{0}} \frac{3\left(\vec{p} \cdot \widehat{e}_{r}\right) \widehat{e}_{r}-\vec{p}}{r^{3}}
$$

Once $\vec{E}_{t i p, a d}^{e x t}$ are calculated, the combination of Eqs. (A.12), (A.11) and (A.10), allows the derivation of a linear set of equations for the $x$ and $z$ components of $\vec{p}_{\text {tip }}$ and $\vec{p}_{a d}$.

Because SRE forces have a very narrow lateral extension, the calculation of the dipolar fields is performed by assuming a planar geometry of the tip-surface interface, which means we assume $R \gg z$. Hence, due to the effect of the image dipoles process, the planar electrodes become antisymmetrical planes. Since $\vec{p}_{t i p}$ belongs to the tip apex plane, it is oriented perpendicularly to this plane, that is, along the $z$ axis. As discussed in the text, we group $\vec{p}_{\text {tip }}$ and its image with regard to the tip plane, which ultimately implies to replace $p_{t i p, z}$ with $2 p_{t i p, z}$ in the calculations. Conversely, $\vec{p}_{\text {ad }}$ cannot be projected along a single direction.

Calculating $\vec{E}_{t i p}^{d i p}$ and $\vec{E}_{a d}^{d i p}$ by using the image dipoles process leads formally to an infinity of image dipoles of $\vec{p}_{t i p}$ and $\vec{p}_{a d}$. Nevertheless, because the dipolar field decreases as $r^{-3}$ [Eq. (A.12)] and because the distance between each image dipole and the real dipole 
increases as the number of images under consideration increases, we restrict the calculation of the dipolar field to the image dipoles depicted in Fig. 5. This configuration stands for the minimum set of images that ensures the antisymmetry condition on the tip apex as well as the coupling between $\vec{p}_{\text {tip }}$ and $\vec{p}_{\text {ad }}$ and their first images. Thus, with this level of approximation, $\vec{E}_{t i p}^{d i p}$ is derived by only considering interactions with $\vec{p}_{a d}^{(0)}$ (real adatom dipole), $\vec{p}_{a d}^{\left(1^{\prime}\right)}, 2 \vec{p}_{t i p}^{(1)}$, and $2 \vec{p}_{\text {tip }}^{\left(2^{\prime}\right)}$ [cf. Fig. (5)]. Similarly, $\vec{E}_{a d}^{d i p}$ is derived by considering interactions with $2 \vec{p}_{\text {tip }}^{(0)}$ (real tip atom dipole), $2 \vec{p}_{t i p}^{(1)}, \vec{p}_{a d}^{(1)}$ and $\vec{p}_{a d}^{\left(1^{\prime}\right)}$.

\section{B. Local electric field felt by the charged adatom: $E_{a d}^{\text {loc }}$}

We seek the expression of the local electric felt by the charged adatom $\vec{E}_{a d}^{l o c}=\vec{E}_{a d}^{e x t}+\vec{E}_{a d}^{d i p}$ [cf. Eq. (A.11)].

(i) The external electric field acting on the charged adatom $\vec{E}_{a d}^{\text {ext }}$ consists of two components: (a) the field of the void capacitor that may locally be assumed as a planar capacitor $(R \gg z)$, referred to as $\vec{E}^{e x t, 0}$, and (b) the field produced by the image charges of the charge within the metallic electrodes of the capacitor, that is the spherical apex of the tip and the counter-electrode, referred to as $\vec{E}_{i m \rightarrow a d}^{e x t}$,

$$
\vec{E}_{a d}^{e x t}=\vec{E}^{e x t, 0}+\vec{E}_{i m \rightarrow a d}^{e x t}
$$

When assuming that the void capacitor has a planar geometry, the expression of the electric field becomes trivial:

$$
\vec{E}^{e x t, 0}=\frac{V_{b}}{h_{d}+z} \widehat{z}
$$

The electric field produced by the image charges of the charge may be derived by recurrence,

$$
\vec{E}_{i m \rightarrow a d}^{e x t}=\frac{q}{4 \pi \epsilon_{e f f}} \sum_{n=0}^{\infty}\left[\frac{1}{\left[2 z+2 d_{n}\right]^{2}}-\frac{1}{\left[2 h_{d}+2 d_{n}\right]^{2}}\right] \widehat{z},
$$

with $d_{n}=n\left(h_{d}+z\right)$ and $n \in \mathbb{N}$. Therefore, $\vec{E}_{a d}^{e x t}$ has a single vertical component. When considering both the convergence of the series and the computing time for the calculations, it is found that $n=20$ is an optimal choice for shrinking the series.

(ii) $\vec{E}_{a d}^{d i p}$ states for the dipolar field influencing the surface adatom. The vectorial projection along $x$ and $z$ axes gives: 


$$
\vec{E}_{a d}^{d i p}=\frac{1}{4 \pi \epsilon_{0}}\left\{\begin{array}{c}
\frac{6 p_{t i p, z}}{r^{3}} \cos \theta \sin \theta-\frac{6 p_{t i p, z}}{r^{\prime 3}} \cos \theta^{\prime} \sin \theta^{\prime}+2 p_{a d, x}\left(\frac{1}{(2 x)^{3}}+\frac{1}{(2 z)^{3}}\right) \\
\frac{2 p_{t i p, z}}{r^{3}}\left(3 \cos ^{2} \theta-1\right)+\frac{2 p_{t i p, z}}{r^{\prime 3}}\left(3 \cos ^{2} \theta^{\prime}-1\right)+2 p_{a d, z}\left(\frac{1}{\left(2 h_{d}\right)^{3}}+\frac{1}{(2 z)^{3}}\right) .
\end{array}\right.
$$

Therefore, the equation giving the expression of the dipolar moment of the adatom becomes:

$$
\vec{p}_{a d}=\epsilon_{0} \alpha \vec{E}_{a d}^{l o c}=\epsilon_{0} \alpha\left\{\begin{array}{c}
E_{a d}^{d i p}\left(p_{t i p, z} ; p_{a d, x}\right) \\
E_{a d}^{e x t}+E_{a d}^{d i p}\left(p_{t i p, z} ; p_{a d, z}\right)
\end{array}\right.
$$

As expected, the dipole of the polarized adatom has two vectorial components. The $x$ component may be derived and put in a simple shape, that is at lowest order in $\alpha$. We get:

$$
p_{a d, x}=\frac{\alpha}{4 \pi} 6 p_{t i p, z}\left(\frac{\cos \theta \sin \theta}{r^{3}}-\frac{\cos \theta^{\prime} \sin \theta^{\prime}}{r^{\prime 3}}\right)
$$

Therefore, it may be seen that the $x$ component of the dipole of the polarized adatom stems from the $z$ (i.e. vertical) component of the tip dipole.

\section{Local electric field felt by the tip atom: $E_{\text {tip }}^{\text {loc }}$}

We now seek the expression of the local electric felt by the tip atom: $\vec{E}_{t i p}^{l o c}=\vec{E}_{t i p}^{e x t}+\vec{E}_{t i p}^{d i p}$.

(i) Contrary to $\vec{E}_{\text {ad }}^{\text {ext }}, \vec{E}_{\text {tip }}^{\text {ext }}$ consists of three components: (a) the field of the void capacitor $\vec{E}^{e x t, 0}$ already found, (b) the field of the image charges of the charge within the metallic electrodes of the capacitor, and (c) the Coulombic field due to the charged adatom. However, the two latter components are derived in a row and give the expression of the electric field referred to as $\vec{E}_{a d+i m \rightarrow t i p}^{e x t}$,

$$
\vec{E}_{t i p}^{e x t}=\vec{E}^{e x t, 0}+\vec{E}_{a d+i m \rightarrow t i p}^{e x t}
$$

We get

$$
\vec{E}_{a d+i m \rightarrow t i p}^{e x t}=\frac{2 q}{4 \pi \epsilon_{e f f}} \sum_{n=0}^{\infty}\left[\frac{z+2 d_{n}}{\left[(z \tan \theta)^{2}+\left(z+2 d_{n}\right)^{2}\right]^{3 / 2}}-\frac{2 h_{d}+z+2 d_{n}}{\left[(z \tan \theta)^{2}+\left(2 h_{d}+z+2 d_{n}\right)^{2}\right]^{3 / 2}}\right] \widehat{z}
$$

with $d_{n}=n\left(h_{d}+z\right)$. For the same reason as before, the calculations are performed with $n=20$. Contrary to $\vec{E}_{i m \rightarrow a d}^{e x t}, \vec{E}_{a d+i m \rightarrow t i p}^{e x t}$ is not zero for $n=0$, which actually gives the Coulombic electric field produced by the charge influencing the tip. 
(ii) $\vec{E}_{t i p}^{d i p}$ states for the dipolar field influencing the tip atom. We get:

$$
\vec{E}_{t i p}^{d i p}=\frac{1}{4 \pi \epsilon_{0}}\left[\frac{4 p_{t i p, z}}{\left(2\left(h_{d}+z\right)\right)^{3}}+\frac{6}{r^{3}}\left(p_{a d, x} \cos \theta \sin \theta+p_{a d, z}\left(\cos ^{2} \theta-1\right)\right)\right] \widehat{z}
$$

Therefore, the second equation giving the expression of the dipolar moment of the tip atom is:

$$
\vec{p}_{t i p}=\epsilon_{0} \alpha \vec{E}_{t i p}^{l o c}=\epsilon_{0} \alpha\left\{\begin{array}{c}
0 \\
E^{e x t, 0}+E_{a d+i m \rightarrow t i p}^{e x t}+E_{t i p}^{d i p}\left(p_{t i p, z} ; p_{a d, x} ; p_{a d, z}\right)
\end{array}\right.
$$

The tip dipole has solely a vertical component, as expected from the symmetry conditions. The set of linear equations (A.17) and (A.22) gives the expressions of $p_{a d, z}$ and $p_{t i p, z}$, which ultimately gives the expression of $F_{S R}^{e l}$ [right-hand side in Eq. (5)].

\section{References}

1 G. Binnig, C. Quate, and C. Gerber, Phys. Rev. Lett. 56, 930 (1986).

2 Y. Martin, C. Williams, and H. Wickramasinghe, J. Appl. Phys. 61, 4723 (1987).

3 T. Albrecht, P. Grütter, D. Horne, and D. Rugar, J. Appl. Phys. 69, 668 (1991).

4 F. J. Giessibl, Science 267, 1451 (1995).

5 in Proceedings of the International Workshop on Noncontact Atomic Force Microscopy, Vol. 140 of Appl. Surf. Sci., edited by S. Morita (Elsevier, Amsterdam, 1999).

6 Y. Sugimoto, P. Pou, M. Abe, P. Jelinek, R. Pérez, S. Morita, and O. Custance, Nature 446, 64 (2007).

7 F. J. Giessibl, H. Bielefeldt, S. Hembacher, and J. Mannhart, Ann. Phys. (Leipzig) 10, 887 (2001).

8 F. J. Giessibl, S. Hembacher, H. Bielefeldt, and J. Mannhart, Science 289, 422 (2000).

9 M. A. Lantz, H. J. Hug, R. Hoffmann, S. Martin, A. Baratoff, and H.-J. Güntherodt, Phys. Rev. B 68, 035324 (2003).

10 Ch. Loppacher, R. Bennewitz, O. Pfeiffer, M. Guggisberg, M. Bammerlin, S. Schär, V. Barwich, A. Baratoff, and E. Meyer, Phys. Rev. B 62, 13674 (2000). 
11 T. König, G. H. Simon, H.-P. Rust, and M. Heyde, Appl. Phys. Lett. 95, 083116 (2009).

12 P. Dieska, I. Stich, and R. Pérez, Phys. Rev. Lett. 91, 216401 (2003).

13 F. J. Giessibl, Phys. Rev. B 45, 13815 (1992).

14 A. Livshits and A. Shluger, Phys. Rev. B 59, 2436 (1999).

15 A. Livshits and A. Shluger, Appl. Surf. Sci. 141, 274 (1999).

16 A. Livshits, A. Shluger, and A. Rohl, Appl. Surf. Sci. 140, 327 (1999).

17 A. S. Foster, C. Barth, A. L. Shluger, R. M. Nieminen, and M. Reichling, Phys. Rev. B 66, $235417(2002)$.

18 A. S. Foster, A. Y. Gal, J. M. Airaksinen, O. H. Pakarinen, and Y. J. Lee, J. D. Gale, A. L. Shluger, and R. M. Nieminen, Phys. Rev. B 68, 195420 (2003).

19 M. A. Lantz, R. Hoffmann, A. S. Foster, A. Baratoff, H. J. Hug, H.-R. Hidber, and H.-J. Güntherodt, Phys. Rev. B 74, 245426 (2006).

20 R. Hoffmann, D. Weiner, A. Schirmeisen, and A. Foster, Phys. Rev. B 80, 115426 (2009).

21 M. Nonnenmacher, M. O’Boyle, and H. Wickramasinghe, Appl. Phys. Lett. 58, 2921 (1991).

22 J. Weaver and D. Abraham, J. Vac. Sci. Technol. B 9, 1559 (1991).

23 S. Hudlet, M. Saint-Jean, C. Guthmann, and J. Berger, Eur. Phys. J. B 2, 5 (1998).

24 M. Saint-Jean, S. Hudlet, C. Guthmann, and J. Berger, J. Appl. Phys. 86, 5245 (1999).

25 B. Law and F. Rieutord, Phys. Rev. B 66, 35402 (2002).

26 S. Sadewasser and M. Ch. Lux-Steiner, Phys. Rev. Lett. 91, 266101 (2003).

27 S. Sadewasser, Th. Glatzel, R. Shikler, Y. Rosenwaks, and M. Ch. Lux-Steiner, Appl. Surf. Sci. 210, $32(2003)$.

28 S. Sadewasser, P. Carl, Th. Glatzel, and M. Ch. Lux-Steiner, Nanotechnology 15, S14 (2004).

29 J. Polesel-Maris, A. Piednoir, T. Zambelli, X. Bouju, and S. Gauthier, Nanotechnology 15, S24 (2004).

30 A. Kikukawa, S. Hosaka, and R. Imura, Rev. Sci. Instrum. 67, 1463 (1996).

31 S. Kitamura and M. Iwatsuki, Appl. Phys. Lett. 72, 3154 (1998).

32 Ch. Sommerhalter, Th. W. Matthes, Th. Glatzel, A. Jäger-Waldau, and M. Ch. Lux-Steiner , Appl. Phys. Lett. 75, 286 (1999).

33 Y. Rosenwaks, R. Shikler, Th. Glatzel, and S. Sadewasser, Phys. Rev. B 70, 085320 (2004).

34 H. Hoppe, Th. Glatzel, M. Niggemann, A. Hinsch, M. Ch. Lux-Steiner, and N. S. Sariciftci, Nanoletters 5, 269 (2004). 
37 Th. Glatzel, S. Sadewasser, and M. Ch. Lux-Steiner, Appl. Surf. Sci. 210, 84 (2003).

38 A. Gil, J. Colchero, J. Gomez-Herrero, and A. Baro, Nanotechnology 14, 332 (2003).

39 U. Zerweck, Ch. Loppacher, T. Otto, S. Grafström, and L. M. Eng, Phys. Rev. B 71, 125424 $(2005)$

40 K. Sajewicz, F. Krok, and J. Konior, Jap. J. Appl. Phys. 49, 025201 (2010).

41 A. Sasahara, C. L. Pang, and H. Onishi, J. Phys. Chem. B 110, 13453 (2006).

42 G. H. Enevoldsen, Th. Glatzel, M. C. Christensen, J. V. Lauritsen, and F. Besenbacher, Phys. Rev. Lett. 100, 236104 (2008).

43 F. Bocquet, L. Nony, Ch. Loppacher, and Th. Glatzel, Phys. Rev. B 78, 035410 (2008).

44 S. Kitamura, K. Suzuki, and M. Iwatsuki, Appl. Surf. Sci. 140, 265 (1999).

45 S. Kitamura, K. Suzuki, M. Iwatsuki, and C. Mooney, Appl. Surf. Sci. 157, 222 (2000).

46 T. Arai and M. Tomitori, Phys. Rev. Lett. 93, 256101 (2004).

47 S. Sadewasser, P. Jelinek, C.-K. Fang, O. Custance, Y. Yamada, Y. Sugimoto, M. Abe, and S. Morita, Phys. Rev. Lett. 103, 266103 (2009).

48 K. Okamoto, K. Yoshimoto, Y. Sugawara, and S. Morita, Appl. Surf. Sci. 210, 128 (2003).

49 Y. Sugawara, T. Uchihashi, M. Abe, and S. Morita, Appl. Surf. Sci. 140, 371 (1999).

50 F. Krok, K. Sajewicz, J. Konior, M. Goryl, P. Piatkowski, and M. Szymonski, Phys. Rev. B 77, $235427(2008)$.

51 K. Okamoto, Y. Sugawara, and S. Morita, Jpn. J. Appl. Phys. 42, 7163-7168 (2003).

52 L. Nony, F. Bocquet, Ch. Loppacher, and Th. Glatzel, Nanotechnology 20, 264014 (2009).

53 L. Nony, A. Foster, F. Bocquet, and Ch. Loppacher, Phys. Rev. Lett. 103, 036802 (2009).

54 M. Bieletzki, T. Hynninen, T. M. Soini, M. Pivetta, C. R. Henry, A. S. Foster, F. Esch, C. Barth, and U. Heiza, Phys. Chem. Chem. Phys. 12, 3203 (2010).

55 A. Masago, M. Tsukada, and M. Shimizu, Phys. Rev. B. 82, 195433 (2010).

56 L. Gross, F. Mohn, P. Liljeroth, J. Repp, F. J. Giessibl, G. Meyer, Science 324, 1428 (2009).

57 B. Terris, J. Stern, D. Rugar, and H. Mamin, Phys. Rev. Lett. 63, 2669 (1989).

58 Ch. Schönenberger and S. Alvarado, Phys. Rev. Lett. 65, 3162 (1990).

59 E. Bussmann, N. Zheng, and C. Williams, Appl. Phys. Lett. 86, 163109 (2005).

60 Y. Azuma, M. Kanehara, T. Teranishi, and Y. Majima, Phys. Rev. Lett. 96, 016108 (2006). 
61 R. Stomp, Y. Miyahara, S. Schär, Q. Sun, H. Guo, and P. Grütter, Phys. Rev. Lett. 94, 056802 (2005).

62 T. D. Krauss and L. E. Brus, Phys. Rev. Lett. 83, 4840 (1999).

63 T. Mélin, H. Diesinger, D. Deresmes, and D. Stiévenard, Phys. Rev. Lett. 92, 166101 (2004).

64 T. Mélin, H. Diesinger, D. Deresmes, and D. Stiévenard, Phys. Rev. B 69, 035321 (2004).

65 T. König, G. H. Simon, H.-P. Rust, G. Pacchioni, M. Heyde, and H.-J. Freund, J. Am. Chem. Soc. 131, $17544(2009)$.

66 J. Israelachvili, Intermolecular and Surface Forces, $2^{\text {nd }} e d$. (Academic Press, New York, 1992), Chap. 11.

67 M. Guggisberg, M. Bammerlin, Ch. Loppacher, O. Pfeiffer, A. Abdurixit, V. Barwich, R. Bennewitz, A. Baratoff, E. Meyer, and H.-J. Güntherodt, Phys. Rev. B 61, 11151 (2000).

68 J. Silberbach, Chem. Phys. Lett. 133, 267 (1987).

69 Magnetic forces are not addressed in this work.

70 L. Kantorovich, A. Foster, A. Shluger, and A. Stoneham, Surf. Sci. 445, 283 (2000).

71 A. Foster, Ph.D. thesis, University College London, 2000.

72 J. Jackson, Classical Electrodynamics, $3^{\text {rd }}$ ed. (J. Wiley and Sons, New York, 1998).

73 A similar argument had been used in the model described in Ref.[43].

74 R. Watson, J. Davenport, M. Perlam, and T. Sham., Phys. Rev. B 24, 1791 (1981).

75 C. Kittel, Introduction to Solid State Physics, $8^{\text {th }}$ ed. (J. Wiley and Sons, 2004).

76 V. Arunachalam, W. H. Marlow, and J. X. Lu, Phys. Rev. E 58, 3451 (1998).

77 J. Repp, G. Meyer, F. E. Olsson, and M. Persson, Science 305, 493 (2004).

78 F. J. Giessibl, Phys. Rev. B 56, 16010 (1997).

79 F. J. Giessibl, Appl. Phys. Lett. 78, 123 (2001).

80 G. Sacha, E. Sahagún, and J. Sáenz, J. Appl. Phys. 101, 024310 (2007).

81 G. Sacha, Appl. Phys. Lett. 97, 033115 (2010).

82 Y. Shen, D. Barnett, and P. Pinsky, Engineering Analysis with Boundary Elements 32, 682-691 (2008). 